\title{
Electron multiplication in a gas discharge at high values of $E / p$
}

\author{
L Friedland \\ Racah Institute of Physics, Hebrew University of Jerusalem, Jerusalem, Israel
}

Received 4 December 1973 in final form 3 June 1974

\begin{abstract}
This paper presents a theoretical study of the electron multiplication in Townsend dis charge in the case of large values of $E / p$. An equation for the electron current density at different discharge points is found. An analytical expression for the first Townsend's ionization coefficient $\alpha / p$ has been obtained from the solution of this equation for a uniform electric field. The slopes $a$ and $k$ of the curves of ionization and total inelastic collision cross sections as functions of energy for some gases are obtained from the analysis of experimental data for $\alpha / p$. Using the values of $a$ and $k$ one can solve the equation for the electron current density also for the case of non-uniform electric field. As an example, electron current densities in the discharge in nitrogen between two concentric cylinders as functions of voltages are calculated.
\end{abstract}

\section{Introduction}

Avalanche ionizations in Townsend discharge in a uniform electric field have been fairly well investigated experimentally over a wide tange of the values of reduced electric field $E / p$ (see for example Loeb 1955). However, theoretical interpretation of experimental data is limited to small values of $E / p$. The reason for this is that in strong electric fields the electron energy distribution loses its spherical symmetry and local character. This problem was first investigated by Morton (1946) who developed a complicated theory to explain experimental results on electron current component growth in electric fields with large space gradients.

More recently, experimental data (Haydon and Stock 1966, Folkard and Haydon 1971) and Monte Carlo calculations (Folkard and Haydon 1970) of the growth of ionization in molecular hydrogen have been published. These investigations show that in a uniform electric field at high values of $E / p$ the spatial growth of ionization cannot be explained by well-accepted equilibrium theory.

This paper presents a general theoretical approach by which the problem of electron current growth at high values of $E / p$ can be reasonably dealt with.

Let $j_{0}$ be the density of electron current from the cathode. As a result of ionization the electron current density in the discharge is a function of the distance $x$ from the cathode:

$$
j(x)=j_{0} N(x)
$$

In $\$ 2$ we consider the general case of non-uniform electric field and construct the equation for the function $N(x)$. 
In the case of small currents, for the uniform electric field between plane electrodes, $N(x)$ is given by

where

$$
N(x)=\exp \left[\alpha\left(x-x_{0}\right)\right]
$$

$$
\frac{\alpha}{p}=\frac{1}{p\left(x-x_{0}\right)} \ln \left(\frac{j(x)}{j_{0}}\right)
$$

is Townsend's first ionization coefficient, $p$ being the pressure of the gas and $x_{0}$ a distance introduced to take into account the non-equilibrium effects at small separations. Section 3.1 deals with the electron multiplication in uniform electric field. We obtain an analytical expression for $\alpha / p$ and, using this expression, analyse the experimental data for $\alpha / p$ for some gases.

If the electric field in a discharge is a function of the distance $x$ from the cathode, $E=E(x)$, then $N(x)$ is often given by

$$
N(x)=\exp \left(\int_{0}^{x} \alpha(x) \mathrm{d} x\right)
$$

where $\alpha(x) / p$ is now Townsend's first ionization coefficient in the electric field $E(x)$. However, Morton (1946) showed that in electric fields with large space gradients the use of the Townsend integral $\int_{0}^{x} \alpha(z) \mathrm{d} z$ is invalid. The reason is that the mean velocity of electrons at any point $x$ in these fields is not equal to the mean velocity of the electrons in the equivalent uniform field. This phenomenon will be illustrated in $\$ 3.2$ for the case of the discharge in nitrogen between two concentric cylinders.

\section{Formal approach}

Suppose that the electric field $E(x)$ is intense enough at every point $x$ of the discharge for all electrons in the avalanche to move along the field lines. We shall assume that the new electrons in the avalanche are 'born' with zero energy. Then the mean energy $\bar{\epsilon}\left(x^{\prime}, x\right)$ of every new electron at the point $x$ depends only on the point $x^{\prime}$ at which this electron was created. It is also clear that, $\bar{\alpha}\left(x^{\prime}, x\right) \mathrm{d} x$, the mean number of ionizations in the interval $\mathrm{d} x$ that makes the electron, which was 'born' at the point $x^{\prime}$, depends also only on $x^{\prime}$ and $x$.

Let us now define $n\left(x^{\prime}\right) \mathrm{d} x^{\prime}$ as the mean number of electrons of the avalanche which were created in the interval $\mathrm{d} x^{\prime}$. Then,

$$
n(x) \mathrm{d} x=\mathrm{d} x \int_{0}^{x} n\left(x^{\prime}\right) \bar{\alpha}\left(x^{\prime}, x\right) \mathrm{d} x^{\prime}+\bar{\alpha}(0, x) \mathrm{d} x .
$$

By definition, $n(x)=\mathrm{d} N(x) / \mathrm{d} x$, where $N(x)$ is the mean number of electrons in the avalanche at the point $x$. Therefore according to (5)

$$
\frac{\mathrm{d} N(x)}{\mathrm{d} x}=\left.N\left(x^{\prime}\right) \bar{\alpha}\left(x^{\prime}, x\right)\right|_{x^{\prime}=0} ^{x^{\prime}=x}-\int_{0}^{x} N\left(x^{\prime}\right) \frac{\partial \bar{x}\left(x^{\prime}, x\right)}{\partial x^{\prime}} \mathrm{d} x^{\prime}+\bar{\alpha}(0, x) .
$$

Putting $N(0)=1$ and $\bar{\alpha}(x, x)=0$ in the last equation gives

$$
\frac{\mathrm{d} N(x)}{\mathrm{d} x}=-\int_{0}^{x} N\left(x^{\prime}\right) \frac{\partial \bar{\alpha}\left(x^{\prime}, x\right)}{\partial x^{\prime}} \mathrm{d} x^{\prime} .
$$

The integral equation (6) yields $N(x)$ if the function $\bar{\alpha}\left(x^{\prime}, x\right)$ is known. One can derive an analytical expression for $\bar{\alpha}\left(x^{\prime}, x\right)$ if the following assumptions are made: in every 
inelastic collision the electron loses the same energy $\xi$, and the dependence of the total inelastic collision efficiency is given by the formulae:

$$
\left.\begin{array}{l}
Q(\epsilon)=0 \quad 0 \leqslant \epsilon \leqslant \xi \\
Q(\epsilon)=p_{0} k(\epsilon-\xi) \quad \xi<\epsilon \quad
\end{array}\right\}
$$

where $p_{0}$ is the pressure reduced to $0^{\circ} \mathrm{C}$.

The energy of the electron (in $\mathrm{eV}$ ) at the point $x$ is now defined only by the number $n$ of inelastic collisions between the creation point $x^{\prime}$ and $x$ :

$$
\epsilon_{n}\left(x^{\prime}, x\right)=\int_{x^{\prime}}^{x} E\left(x^{\prime \prime}\right) \mathrm{d} x^{\prime \prime}-n \xi .
$$

Thus, the mean energy

$$
\bar{\epsilon}\left(x^{\prime}, x\right)=\sum_{n} f_{n}\left(x^{\prime}, x\right) \epsilon_{n}\left(x^{\prime}, x\right)=\int_{0}^{x} E\left(x^{\prime \prime}\right) \mathrm{d} x^{\prime \prime}-\bar{n}\left(x^{\prime}, x\right) \xi
$$

where $f_{n}\left(x^{\prime}, x\right)$ is the probability that $n$ collisions will occur and $n\left(x^{\prime}, x\right)=\sum_{n} n f_{n}\left(x^{\prime}, x\right)$. By differentiation:

$$
\frac{\partial \bar{\epsilon}\left(x^{\prime}, x\right)}{\partial x}=E(x)-\frac{\partial \bar{n}\left(x^{\prime}, x\right)}{\partial x} .
$$

$\left[\partial \bar{n}\left(x^{\prime}, x\right) / \partial x\right] \mathrm{d} x$ is the mean number of inelastic collisions in the interval $\mathrm{d} x$, and therefore

$$
\frac{\partial \bar{n}\left(x^{\prime}, x\right)}{\partial x}=\sum_{n} Q\left(\epsilon_{n}\left(x^{\prime}, x\right)\right) f_{n}\left(x^{\prime}, x\right)=\bar{Q}\left(x^{\prime}, x\right) .
$$

Thus from (10)

$$
\frac{\partial \bar{\epsilon}\left(x^{\prime}, x\right)}{\partial x}=E(x)-\bar{Q}\left(x^{\prime}, x\right) \xi
$$

The energy $\epsilon_{n}\left(x^{\prime}, x\right)$ is always greater than zero; therefore, for every $x$ and $x^{\prime}$ there is an integer $m$ such that

$$
\begin{array}{lll}
f_{m+i}\left(x^{\prime}, x\right)=0, & i=0,1,2, \ldots & \\
f_{m-1}\left(x^{\prime}, x\right) \neq 0, & Q\left(\epsilon_{m-1}\left(x^{\prime}, x\right)\right)=0, & 0 \leqslant \epsilon_{m-1}\left(x^{\prime}, x\right)<\xi \\
f_{m-2}\left(x^{\prime}, x\right) \neq 0, & Q\left(\epsilon_{m-2}\left(x^{\prime}, x\right)\right) \neq 0, & \xi \leqslant \epsilon_{m-2}\left(x^{\prime}, x\right)<2 \xi
\end{array}
$$

Thus there will be a finite number of the terms in the sum (11):

$$
\bar{Q}\left(x^{\prime}, x\right)=p_{0} k\left(\epsilon_{0}-\xi\right) f_{0}+\ldots+p_{0} k\left(\epsilon_{m-2}-\xi\right) f_{m-2} .
$$

By simple algebra

where

$$
\bar{Q}\left(x^{\prime}, x\right)=p_{0} k\left(\bar{\epsilon}\left(x^{\prime}, x\right)-\xi\right)+p_{0} k \Delta\left(x^{\prime}, x\right)
$$

$$
\Delta\left(x^{\prime}, x\right)=\left(\xi-\epsilon_{m-1}\right) f_{m-1} .
$$

In (14) $0 \leqslant f_{m-1}\left(x^{\prime}, x\right) \leqslant 1$ and $0 \leqslant \epsilon_{m-1} \leqslant \xi$; therefore

$$
0 \leqslant \Delta\left(x^{\prime}, x\right) \leqslant \xi \text {. }
$$


Putting expression (13) into (12), we get

$$
\frac{\partial \bar{\epsilon}\left(x^{\prime}, x\right)}{\partial x}=E(x)-p_{0} k \xi\left(\bar{\epsilon}\left(x^{\prime}, x\right)-\xi+\Delta\left(x^{\prime}, x\right)\right) .
$$

The solution of this equation (with $\epsilon\left(x^{\prime}, x^{\prime}\right)=0$ ) is

$$
\bar{\epsilon}\left(x^{\prime}, x\right)=\exp \left(-p_{0} k \xi x\right) \int_{x^{\prime}}^{x}\left[E\left(x^{\prime \prime}\right)+p_{0} k \xi\left(\xi-\Delta\left(x^{\prime}, x^{\prime \prime}\right)\right)\right] \exp \left(p_{0} k \xi x^{\prime \prime}\right) \mathrm{d} x^{\prime \prime}
$$

From (15), $0 \leqslant \xi-\Delta\left(x^{\prime}, x\right) \leqslant \xi$. If the electric field $E(x)$ is intense enough, and the inequality

$$
E(x) / p_{0} \gg k \xi^{2}
$$

holds for every point $x$, then approximately

$$
\bar{\epsilon}\left(x^{\prime}, x\right)=\exp \left(-p_{0} k \xi x\right) \int_{x^{\prime}}^{x} E\left(x^{\prime \prime}\right) \exp \left(p_{0} k \xi x^{\prime \prime}\right) \mathrm{d} x^{\prime \prime} .
$$

Let $Q_{i}(\epsilon)$ be the efficiency of ionization of the gas; then

$$
\bar{\alpha}\left(x^{\prime}, x\right)=\bar{Q}_{i}\left(x^{\prime}, x\right) .
$$

Let us assume now that

$$
\left.\begin{array}{l}
Q_{i}(\epsilon)=0 \quad 0 \leqslant \epsilon \leqslant \xi \\
Q_{i}(\epsilon)=p_{0} a(\epsilon-\xi) \quad \xi<\epsilon .
\end{array}\right\}
$$

Then, as in (13), we get

$$
\bar{\alpha}\left(x^{\prime}, x\right)=p_{0} a\left(\tilde{\epsilon}\left(x^{\prime}, x\right)-\xi+\Delta\left(x^{\prime}, x\right)\right)
$$

or, using (16),

$$
\bar{\alpha}\left(x^{\prime}, x\right)=\frac{a}{k \xi}\left(E(x)-\frac{\partial \bar{\epsilon}\left(x^{\prime}, x\right)}{\partial x}\right) .
$$

Putting (19) into (22), we have

$$
\bar{\alpha}\left(x^{\prime}, x\right)=p_{0} a \exp \left(-p_{0} k \xi x\right) \int_{x^{\prime}}^{x} E\left(x^{\prime \prime}\right) \exp \left(p_{0} k \xi x^{\prime \prime}\right) \mathrm{d} x^{\prime \prime} .
$$

Let us once again return to equation (6):

$$
\frac{\mathrm{d} N(x)}{\mathrm{d} x}=-\int_{0}^{x} N\left(x^{\prime}\right) \frac{\partial \bar{\alpha}\left(x^{\prime}, x\right)}{\partial x^{\prime}} \mathrm{d} x^{\prime} .
$$

According to (23) we obtain

$$
\frac{\mathrm{d} N(x)}{\mathrm{d} x}=p_{0} a \exp \left(-p_{0} k \xi x\right) \int_{0}^{x} N\left(x^{\prime}\right) E\left(x^{\prime}\right) \exp \left(p_{0} k \xi x^{\prime}\right) \mathrm{d} x^{\prime} .
$$

We shall again differentiate the last equation:

$\frac{\mathrm{d}^{2} N(x)}{\mathrm{d} x^{2}}=p_{0} \mathrm{a} N(x) E(x)-p_{0} k \xi\left(p_{0} \mathrm{a} \exp \left(-p_{0} k \xi x\right) \int_{0}^{x} N\left(x^{\prime}\right) E\left(x^{\prime}\right) \exp \left(p_{0} k \xi x^{\prime}\right) \mathrm{d} x^{\prime}\right)$, or, using (24),

$$
\frac{\mathrm{d}^{2} N(x)}{\mathrm{d} x^{2}}+p_{0} k \xi \frac{\mathrm{d} N(x)}{\mathrm{d} x}-p_{0} a E(x) N(x)=0 .
$$


This differential equation allows us to define $N(x)$ if the necessary constants and the electric field $E(x)$ in the discharge are known. The boundary conditions for (25) are $N(0)=1$ and $\mathrm{d} N(x) /\left.\mathrm{d} x\right|_{x=0}=0$.

\section{Examples and conclusions}

\subsection{Electron multiplication in uniform fields}

The solution of equation (25) for uniform electric field $E$ and large distances from the cathode can be written as

where

$$
N(x)=\exp \left(\alpha^{\prime} x+\Delta^{\prime}\right)
$$

$$
\alpha^{\prime}=-\frac{p_{0} k \xi}{2}+\left[\left(\frac{p_{0} k \xi}{2}\right)^{2}+a E p_{0}\right]^{1 / 2}
$$

and

$$
\Delta^{\prime}=\ln \left\{\frac{1}{2}\left[1+\left(1+\frac{4 a}{(k \xi)^{2}} \frac{E}{p_{0}}\right)^{-1 / 2}\right]\right\} .
$$

Then the first Townsend's ionization coefficient is given by

$$
\frac{\alpha}{p_{0}}=\frac{\alpha^{\prime}}{p_{0}}=-\frac{k \xi}{2}+\left[\left(\frac{k \xi}{2}\right)^{2}+a \frac{E}{p_{0}}\right]^{1 / 2}
$$

and the length $x_{0}$ (see equation 2 ) by

$$
x_{0}=\Delta^{\prime} / \alpha
$$

It is easily seen that $\alpha / p_{0} \approx(a / k \xi)\left(E / p_{0}\right)$ when $a\left(E / p_{0}\right) \ll(k \xi / 2)^{2}$. Then according to (18), $4 a \ll k$, and that apparently does not exist. When $E / p_{0}$ grows, the function

$$
\alpha / p_{0}=f\left[\left(E / p_{0}\right)^{1 / 2}\right]
$$

tends to its asymptotic form:

$$
\frac{\alpha}{p_{0}}=\left(\frac{a E}{p_{0}}\right)^{1 / 2}-\frac{k \xi}{2}
$$

One of the assumptions made in our theory is that the electrons move in the direction of the electric field. Let us further modify our theory so as to take into account slight variations in the direction of motion of the electrons. Now let us assume that they move at small angle $\theta$ with respect to the direction of the electric field. In this case, all our previously developed equations remain correct if we will introduce $p^{\prime}=p_{0} / \cos \theta$ instead of $p_{0}$. As a first approximation $\theta=C /\left(E / p_{0}\right)$. Then equation (29) with this correction becomes

$$
\frac{\alpha}{p_{0}} \approx-\frac{k \xi}{2}+\left[\left(\frac{k \xi}{2}\right)^{2}-a C+a \frac{E}{p_{0}}\right]^{1 / 2} .
$$

Therefore, if $(k \xi / 2)^{2} \approx a C$, equation (31) will be a good approximation for $\alpha / p_{0}$. A similar relation for $\alpha / p_{0}$ was received experimentally by Posin (1936). He showed that for nitrogen over a large range of $E / p_{0}\left(200-1000 \mathrm{~V} \mathrm{~cm}^{-1}\right.$ Torr $\left.^{-1}\right)$

$$
\left(\frac{\alpha}{p_{0}}+3 \cdot 65\right)^{2}=0 \cdot 21 \frac{E}{p_{0}} \text {. }
$$

Then, according to (31), $a_{\mathrm{N}_{2}}=0.21(\mathrm{~cm} \text { Torr eV) })^{-1}$ and $\frac{1}{2}(k \xi)_{\mathrm{N}_{2}}=3.65(\mathrm{~cm} \mathrm{Torr})^{-1}$. 
The study of the experimental data for $\alpha / p_{0}$ shows that for many gases the graph of the function $\alpha / p_{0}=f\left[\left(E / p_{0}\right)^{1 / 2}\right]$ is a straight line over a wide range $R$ of the values of $E / p_{0}$ : see eg Kruithof and Penning's (1937) data for neon in our figure 1. Thus, from (31) we find that the slope of this line is equal to $a^{1 / 2}$. In addition, the values of $a$ from ionization cross sections are well known experimentally for the majority of the gases. In this way it is possible to test the theory.

From the graphs $\alpha / p_{0}=f\left[\left(E / p_{0}\right)^{1 / 2}\right]$ we can obtain the value of $\frac{1}{2} k \xi$. Taking approximately

$$
\xi=\frac{\xi_{\text {minimum }}+\xi_{\text {ionization }}}{2}
$$

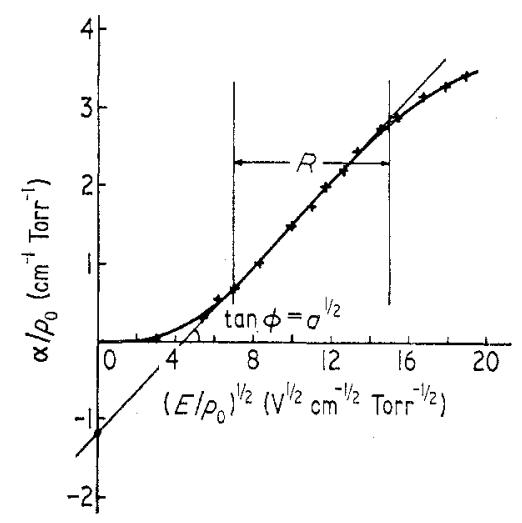

Figure 1. Townsend's first ionization coefficient in neon as a function of $\left(E / p_{0}\right)^{1 / 2}$ (Kruithof and Penning 1937).

Table 1

\begin{tabular}{|c|c|c|c|c|c|}
\hline Gas: & $\mathrm{N}_{2}$ & $\mathrm{H}_{2}$ & $\mathrm{He}$ & $\mathrm{Ne}$ & $\mathrm{Hg}$ \\
\hline $\begin{array}{l}\text { Reference } \\
\text { for } \alpha / p_{0} \text { : }\end{array}$ & $\begin{array}{l}\text { Posin } \\
\text { (1936) }\end{array}$ & $\begin{array}{l}\text { Folkard } \\
\text { and } \\
\text { Haydon } \\
(1971)\end{array}$ & $\begin{array}{l}\text { Druyvesteyn } \\
\text { and } \\
\text { Penning } \\
(1940)\end{array}$ & $\begin{array}{l}\text { Kruithof } \\
\text { and } \\
\text { Penning } \\
(1937)\end{array}$ & $\begin{array}{l}\text { Badareu } \\
\text { and } \\
\text { Bratescu } \\
(1944)\end{array}$ \\
\hline $\begin{array}{l}\text { Range of validity } \\
R\left(\mathrm{~V} \mathrm{~cm}^{-1} \mathrm{Torr}^{-1}\right) \\
\text { from figure } 1:\end{array}$ & $200-1000$ & $50-170$ & $25-100$ & $50-250$ & $200-650$ \\
\hline $\begin{array}{l}a\left(\mathrm{~cm}^{-1} \text { Torr }^{-1} \mathrm{eV}^{-1}\right) \\
\text { from the graphs } \\
\alpha / p_{0}=f\left[\left(E / p_{0}\right)^{1 / 2}\right]:\end{array}$ & $0 \cdot 21$ & 0.13 & 0.032 & 0.072 & 0.77 \\
\hline $\begin{array}{l}a\left(\mathrm{crn}^{-1} \mathrm{Torr}^{-1} \mathrm{eV}^{-1}\right) \\
\text { from cross sections } \\
\text { from von Engel (1965) }\end{array}$ & $: 0.26$ & 0.21 & 0.046 & 0.056 & 0.83 \\
\hline$k \xi\left(\mathrm{cm}^{-1} \operatorname{Torr}^{-1}\right)$ & $7 \cdot 3$ & $4 \cdot 5$ & $1 \cdot 2$ & $2 \cdot 4$ & $17 \cdot 4$ \\
\hline $\begin{array}{l}\xi=\frac{1}{2}\left(\xi \mathrm{min} .+\xi_{\text {ioniz. }}\right)(\mathrm{e} \\
\text { from } \\
\text { von Engel }(1965):\end{array}$ & $\begin{array}{l}\text { V) } \\
11 \cdot 0\end{array}$ & $11 \cdot 2$ & $22 \cdot 2$ & $19 \cdot 2$ & $7 \cdot 62$ \\
\hline$k\left(\mathrm{~cm}^{-1} \operatorname{Torr}^{-1} \mathrm{eV}^{-1}\right)$ & 0.66 & 0.4 & 0.054 & 0.125 & $2 \cdot 28$ \\
\hline
\end{tabular}


we then evaluate the slope of the total inelastic collision cross section $k$. These calculations were made for some gases and the results are presented in table 1 . We find that the values of $a$ derived from the graphs $\alpha / p_{0}=f\left[\left(E / p_{0}\right)^{1 / 2}\right]$ and from ionization cross sections are approximately equal, to within $30 \%$.

\section{2. Ionization in non-uniform electric fields}

For the case of non-uniform electric fields, in (25) we substitute

Then

$$
N(x)=\exp \left(\int_{0}^{x} \alpha(x) \mathrm{d} x\right)
$$

$$
\mathrm{d} \alpha / \mathrm{d} x+\alpha^{2}+p_{0} k \xi \alpha-p_{0} a E(x)=0
$$

where $\alpha(0)=0$. Let us rewrite the last equation in the following form:

$$
\mathrm{d} \alpha / \mathrm{d} x=-\left(\alpha-\alpha_{1}\right)\left(\alpha-\alpha_{2}\right)
$$

where $\alpha_{1}(x)$ and $\alpha_{2}(x)$ are the solutions of the equilibrium equation

$$
\begin{aligned}
& \alpha^{2}+p_{0} k \xi \alpha-p_{0} a E(x)=0 \\
& \frac{\alpha_{1}}{p_{0}}=-\frac{k \xi}{2}+\left[\left(\frac{k \xi}{2}\right)^{2}+a \frac{E(x)}{p_{0}}\right]^{1 / 2} \geqslant 0 \\
& \frac{\alpha_{2}}{p_{0}}=-\frac{k \xi}{2}-\left[\left(\frac{k \xi}{2}\right)^{2}+a \frac{E(x)}{p_{0}}\right]^{1 / 2} \leqslant 0 .
\end{aligned}
$$

We get that

$$
\operatorname{sign}(\mathrm{d} \alpha / \mathrm{d} x)=-\operatorname{sign}\left(\alpha-\alpha_{1}\right)
$$

and, therefore, equation (35) jllustrates the tendency of the function $\alpha(x)$ to reach the equilibrium value $\alpha_{1}$.

It was shown in $\$ 3.1$ that for the case of uniform electric field we obtain a more exact functional dependence of $\alpha_{1} / p_{0}$ on $E / p_{0}$ if we omit the quantity $(k \xi / 2)^{2}$ in equations (37). Assuming the same also in the case of non-uniform electric field and using the known values of $a$ and $k$, one can solve equation (35) if $E / p_{0}$ is within the range $R$ at every point of the discharge gap.

One of the problems in which such a calculation is required is the theoretical determination of the sparking voltage. If, for a given gas pressure and distance between electrodes, the voltage is raised sufficiently, then breakdown should occur when

$$
\gamma[N(d)-1]=1
$$

where $\gamma$ is Townsend's second ionization coefficient near the cathode, and $d$ is the distance between electrodes. Let us take, as an example, the case of a discharge in nitrogen between two concentric cylinders of radius $r_{1}=1 \mathrm{~cm}, r_{2}=2 \mathrm{~cm}$. Let the pressure $p_{0}$ be 1 Torr and the voltage in the range $150-350 \mathrm{~V}$. It is easily seen that for this case the values of $E(r) / p_{0}=V /\left[r \ln \left(r_{2} / r_{1}\right)\right]$ are within $R$. The solution $\alpha^{+}(r)$ of equation (35) when the inner cylinder is the anode is presented in figure $2(a)$; likewise, the solution $\alpha^{-}(r)$ when the inner cylinder is the cathode. The equilibrium function $\alpha_{1}(r)$ is also presented in figure $2(a)$. The values

$$
N^{-}(d)=\exp \left(\int_{r_{1}}^{r_{2}} \alpha^{-}(r) \mathrm{d} r\right), \quad N^{+}(d)=\exp \left(\int_{r_{1}}^{r_{2}} \alpha^{+}(r) \mathrm{d} r\right), \quad \text { and } \quad N_{\operatorname{eq}}(d)=\exp \left(\int_{r_{1}}^{r_{2}} \alpha_{1}(r) \mathrm{d} r\right)
$$



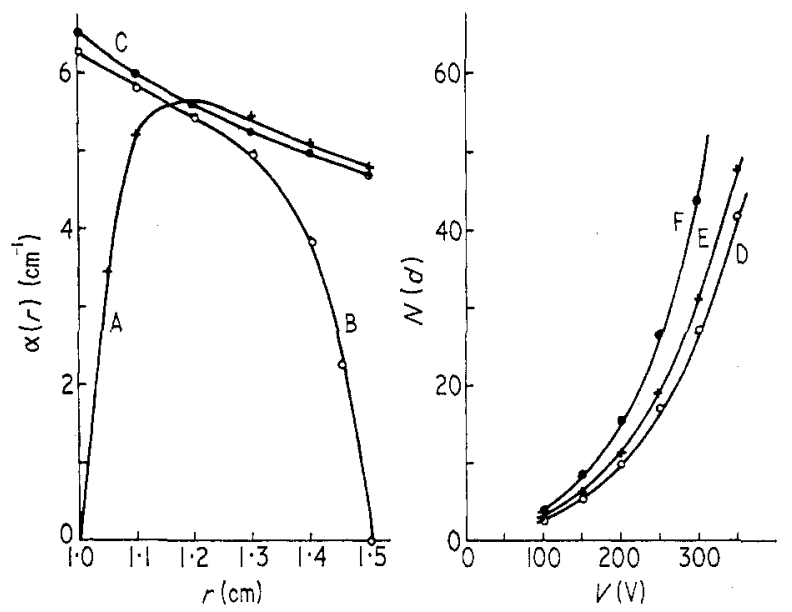

Figure 2. (a) The dependence of the ionization coefficient in nitrogen in cylindrical geometry on the distance $r$ from the axis: A, $\alpha^{-}(r)$, the inner cylinder negative, $V=200 \mathrm{~V}$; $\mathrm{B}, \alpha^{+}(r)$, the inner cylinder positive, $\mathrm{V}=200 \mathrm{~V} ; \mathrm{C}, \alpha_{1}(r), \mathrm{V}=200 \mathrm{~V} .(b)$ Variation of $N(d)=\exp \left(\int_{r_{1}}^{r_{2}} \alpha(r) \mathrm{d} r\right)$ with discharge voltage. D, $N^{+}(d), \alpha(r) \equiv \alpha^{\ddagger}(r) ; \mathrm{E}, \quad N^{-}(d)$, $\alpha(r) \equiv \alpha^{-}(r) ; \mathrm{F}, N_{\mathrm{eq}}(d), \alpha(r) \equiv \alpha_{1}(r)$.

as a function of voltage are presented in figure $2(b)$. The different dependence of $\alpha^{+}(r)$ and $\alpha^{-}(r)$ on $r$ should be noted. $\alpha^{-}(r)$ reaches the equilibrium with the local field (the values of $\alpha_{1}(r)$ ) faster than $\alpha^{+}(r)$. This explains the fact that for all voltages $V$, we get $N^{+}(d)<N^{-}(d)<N_{\text {eq }}(d)$. Thus, according to (38), we conclude that: (a) if we take nonequilibrium ionization (given by equation 35 ) into account, it leads to a bigger theoretical sparking voltage; and $(b)$ the sparking voltage when the inner cylinder is the cathode is smaller than when the inner cylinder is the anode, even if $\gamma$ in both cases is the same.

\section{Acknowledgments}

The author is greatly indebted to Drs P Avivi, F Dothan-Deutsch, and J L Hirshfield for the discussions of the results of this work.

\section{References}

Badareu E and Bratescu G G 1944 Bull. Soc. Romaine Phys. 45 9-13 Druyvesteyn M J and Penning F M 1940 Rev. Mod. Phys. 12 87-174 von Engel A 1965 Ionized Gases (Oxford: Clarendon Press) pp 63-73 Folkard M A and Haydon S C 1970 Aust. J. Phys. 23 847-61

- 1971 Aust. J. Phys. 24 527-42

Haydon S C and Stock H M P 1966 Aust. J. Phys. $19795-803$

Kruithof A A and Penning F M 1937 Physica 4 430-49

Loeb L B 1955 Basic Processes of Gaseous Electronics (Berkeley, Calif.: California UP)

Morton DL 1946 Phys. Rev. 70 358-66

Posin D Q 1936 Phys. Rev. 50 650-58 